\title{
Objets Connectés
}

\section{Mise en œuvre d'un challenge intitulé « Industrie du futur, c'est maintenant ! » dans le cadre d'un enseignement pour des élèves ingénieurs}

\author{
Pascal Vrignat ${ }^{1}$, David Delouche ${ }^{2}$, Manuel Avila ${ }^{1}$, Stéphane Begot ${ }^{1}$, Christophe Bardet $^{1}$, \\ Florent Duculty ${ }^{1}$, Frédéric Kratz ${ }^{3}$ \\ ${ }^{1}$ Université d'Orléans, IUT de l'Indre, Laboratoire PRISME, Châteauroux, France \\ ${ }^{2}$ HEI Campus Centre, Laboratoire PRISME, Châteauroux, France \\ ${ }^{3}$ INSA Centre Val de Loire, Laboratoire PRISME, Bourges, France \\ pascal.vrignat@univ-orleans.fr
}

\begin{abstract}
RESUME : Parmi les nombreuses stratégies pédagogiques permettant d'améliorer la motivation, le savoir-être et le savoir-faire des élèves ou des étudiants, la pédagogie par projet est souvent citée comme exemple. $C$ 'est dans ce contexte, qu'un challenge intitulé "L'industrie du futur, c'est maintenant! " a été activé en 2018 pour des élèves ingénieurs de spécialités mécatronique et robotique. Sept équipes tirées au sort et constituées d'élèves en $5^{\text {ème }}$ année à HEI campus Centre ont donc participé à ce challenge. Les enjeux techniques à franchir étaient vastes car ils devaient répondre à certains besoins et services consacrés aux enjeux liés à l'industrie digitale ou l'industrie 4.0. Un système de production de jetons en polymère pour serrure de caddy et connecté en réseau a servi de support. Cet article détaille la stratégie pédagogique qui a été adoptée, le cahier des charges technique ainsi que la planification à respecter et certains résultats significatifs. Un outil de mesures concernant l'efficience de la méthode a également été mis en place. Les résultats ainsi obtenus nous ont permis d'affiner notre retour sur expérience concernant le challenge.
\end{abstract}

Mots clés : pédagogie par projet, challenge digital, industrie du futur, internet des objets, élèves ingénieurs.

\section{Introduction}

Parmi les nombreuses stratégies pédagogiques permettant d'améliorer la motivation, le savoir-être et le savoir-faire des élèves, la pédagogie par projet est souvent citée comme exemple depuis plusieurs décennies. Devenue une pratique presque quotidienne dans l'enseignement professionnel, elle est de plus en plus utilisée dans l'enseignement supérieur [3] [17], [18].

On peut attribuer l'intérêt de cette pédagogie à deux raisons principales :

This is an Open Access article distributed under the terms of the Creative Commons Attribution License (http://creativecommons.org/licenses/by/4.0), which permits unrestricted use, distribution, and reproduction in any medium, provided the original work is properly cited. 
$\checkmark$ Les compétences des étudiants sont suffisamment développées pour mener des travaux de longue durée dans des contextes où ils sont plus autonomes et où la réponse n'est pas connue forcément à l'avance par l'enseignant.

$\checkmark$ La professionnalisation de l'enseignement supérieur doit être accrue avec notamment, des conventions ou des collaborations avec des industriels.

Une des caractéristiques essentielles des projets étudiants est leur durée, relativement grande, plus longue dans tous les cas que les activités pédagogiques habituelles. Cette durée impose une cohérence dans l'action et dans la réflexion, avec une gestion organisationnelle du groupe ou des groupes de travail. Cependant, il faut être vigilant et anticiper certaines actions pour ne pas sombrer dans certaines dérives (Figure 1). Il existe différents moyens devant être mis en place pour gérer convenablement un projet. On pourra retenir par exemples : le cahier des charges fonctionnel, l'analyse fonctionnelle, les spécifications techniques du besoin, le cycle en V... Notre département de formations à l'Université d'Orléans associé à d'autres formations (HEI campus Centre, INSA Centre Val de Loire) ne sont pas en retard sur ces sujets [2], [22], [24].

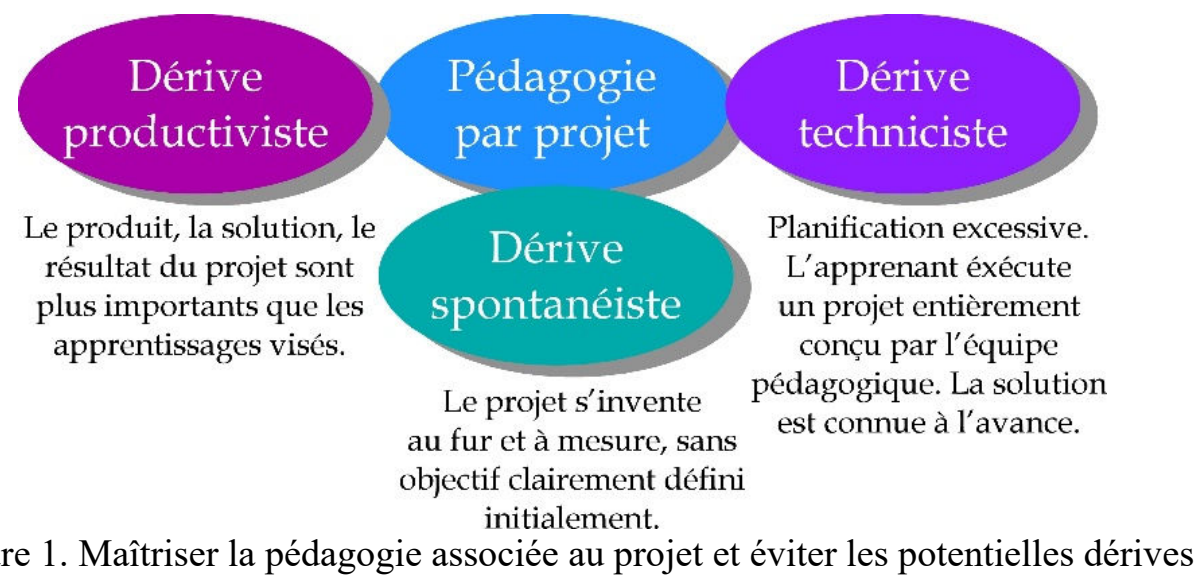

Contrairement à l'horizon des métiers (excepté le passage en retraite pour un salarié), celui des projets est clairement borné par une fin annoncée au préalable. Entre le début et la fin du projet, se déploie un processus d'apprentissage que Midler [13] décrit comme "une dynamique irréversible où l'on passe d'une situation où l'on ne sait rien ou pas grand-chose mais où tout est possible, à une autre où, au contraire, le niveau de connaissances a atteint son maximum mais où toutes les marges de manœuvre ont été utilisées ». La Figure 2 fait une représentation possible de cette situation. Nous avons utilisé un outil moderne de mesures afin de pouvoir tenter de reproduire ce retour sur expérience pédagogique. Cet outil concerne un service disponible sur internet. Son nom est Kahoot [9]. C'est un environnement qui permet d'effectuer en ligne, des Quizz, des sondages ou des QCMs. La section 5 de cet article est consacrée à un Benchmark sur ce sujet. 


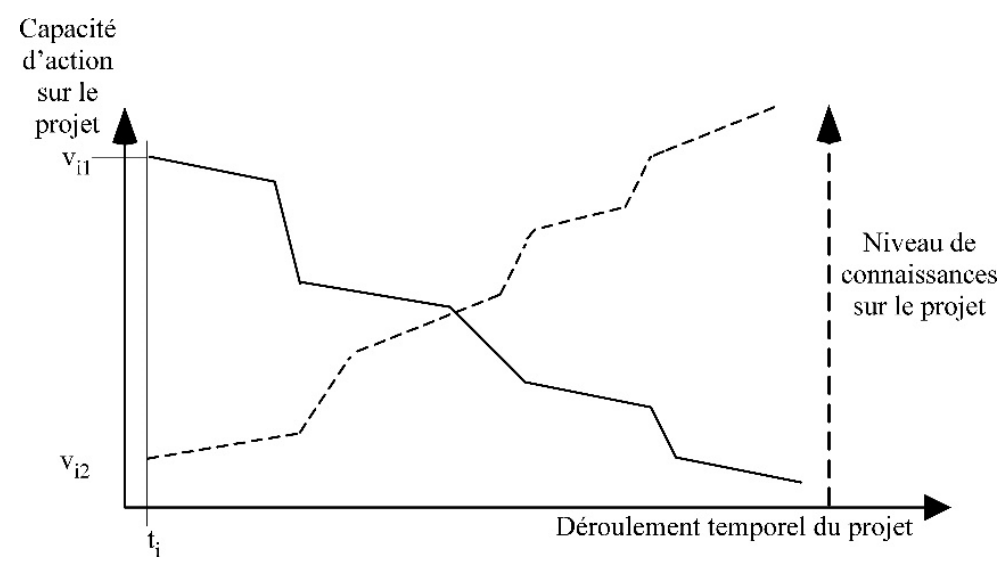

Figure 2. Représentation adaptée de la dynamique de la situation dite «projet » [13]

Enseignants, nous avons également pu remarquer qu'il y a peu d'engouement de la part des apprenants sur un sujet, si le sujet n'est pas à la fois, motivant, attractif et relevant de l'actualité, ludique et démonstratif. Le thème choisi pour cette expérience était de facto, tout trouvé compte tenu, des enjeux industriels actuels. En effet, les filières de formations et de recherche technologiques françaises sont fondamentales pour le développement de l'industrie et de notre pays. Elles doivent attirer vers elles, les meilleurs éléments et former les acteurs utiles au développement des entreprises pour des secteurs d'activités variés tout en leur permettant également d'innover [11], [15]. La France doit jouer un grand rôle dans le développement de l'Industrie du Futur aussi bien au niveau européen que mondial [14]. Notre pays regroupe, en effet, un certain nombre d'entreprises et de startups portées par l'innovation. Processus continu, le développement de l'industrie du futur ne pourra se concrétiser qu'avec l'émergence de nouveaux talents. C'est pour cela que les industriels et l'enseignement supérieur doivent travailler main dans la main. Ces changements profonds dans l'industrie soulèvent également de nombreuses questions liées aux contenus des formations :

$\checkmark$ Quels contenus faut-il développer notamment en termes de compétences ?

$\checkmark$ Sur quels niveaux de formation l'effort doit-il se porter?

$\checkmark$ Comment structurer l'offre de formation?

$\checkmark$ Comment enseigner?

$\checkmark$ Les pédagogies doivent-elles évoluer?

$\checkmark$ Quels sont les équipements et les infrastructures nécessaires pour enseigner ces nouveaux champs disciplinaires?

L'enseignement supérieur technique, technologique et scientifique apporte déjà des éléments de réponse en proposant des formations adéquates sur de nombreuses thématiques liées à l'industrie du futur. Le besoin de ces compétences est cependant tel, qu'il est nécessaire de multiplier rapidement des parcours adaptés de formations.

Cet article est composé de six sections. Après une introduction sur le sujet, la section 2 présente le contexte technique du challenge associé à 2 partenaires industriels. La section 3 décrit la démarche pédagogique de travail qui a été adoptée avec les élèves. La section 4 présente un éventail de résultats significatifs obtenus par différents groupes. La section 5 propose un benchmark pédagogique obtenu à l'issu du challenge. Nous terminons nos propos par une conclusion. 


\section{Le contexte technique du challenge et de la collaboration avec un fabricant de solutions industrielles d'automatisation ainsi qu'un éditeur de logiciel}

\subsection{Une mini-usine comme environnement de production et support du challenge}

Ce challenge nommé « Industrie du futur, c'est maintenant ! » devait s'appuyer sur un contexte réel relevant d'un processus industriel et disponible à l'école d'ingénieurs (Figure 3).

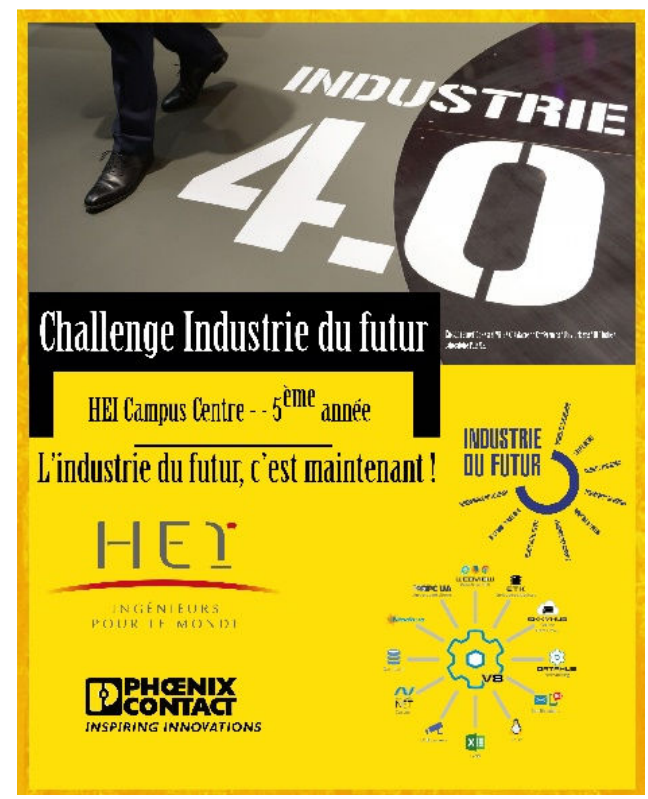

Figure 3. L'affiche du challenge

L'école HEI campus Centre située à Châteauroux dispose depuis quelques mois d'un processus industriel complet (mini-usine). Ce processus est divisé en sous fonctions capables de: produire, contrôler, packager et acheminer jusqu'à une zone logistique, des jetons en polymère (déverrouillage de serrures de chariots que l'on peut trouver sur les aires de grandes surfaces). Ce processus est fabriqué et commercialisé par la société BEMA [8] (Figure 4).
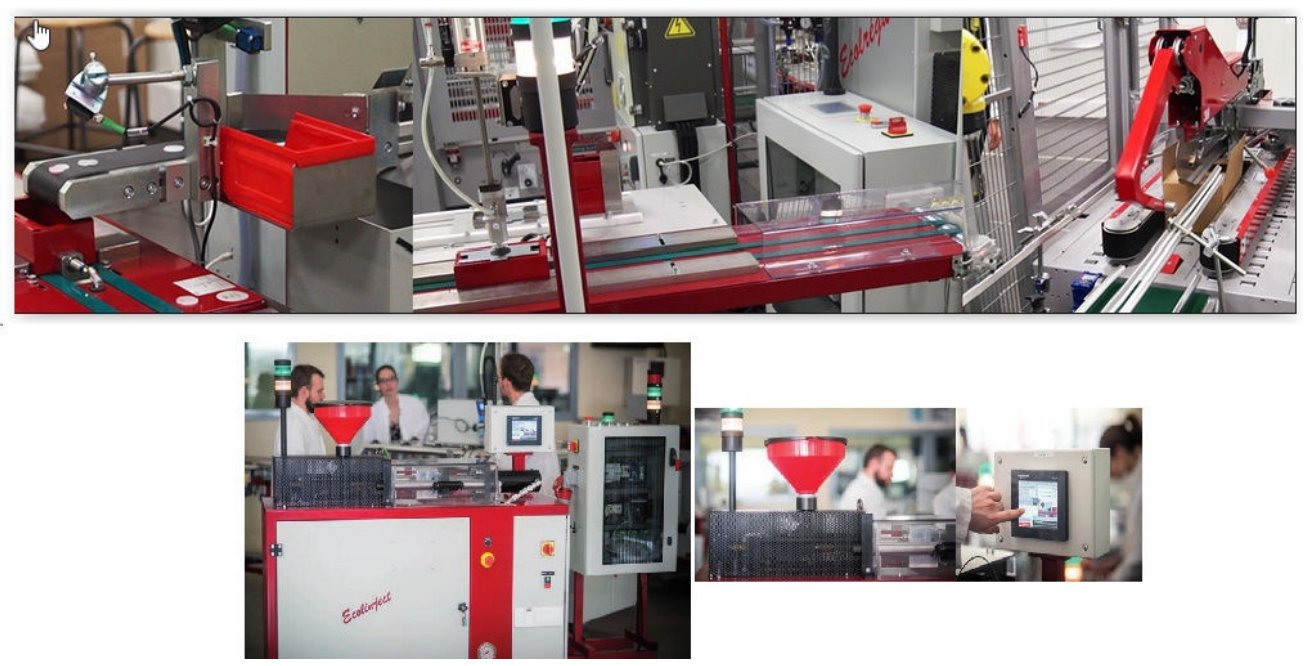

Figure 4. Le processus industriel

Proposition d'article pour le Journal: J3eA, Journal sur l'enseignement des sciences et technologies de l'information et des systèmes 
Ce processus complexe connecté via un réseau de communication numérique est capable de faire coopérer différents sous-ensembles et notamment une zone de manutention robotisée (Robot). Conformément à la Figure 5, il intègre 5 sous-ensembles : Ecolinject (fabrication et contrôle-qualité des jetons), Ecolbox (constitution des boites avec jetons en conformité avec des recettes de fabrication), Ecolregul (filmage individuel des boites), Robot (remplissage de cartons à partir de $\mathrm{n}$ boites), Scotcheuse (fermeture des cartons avant gestion de la logistique).

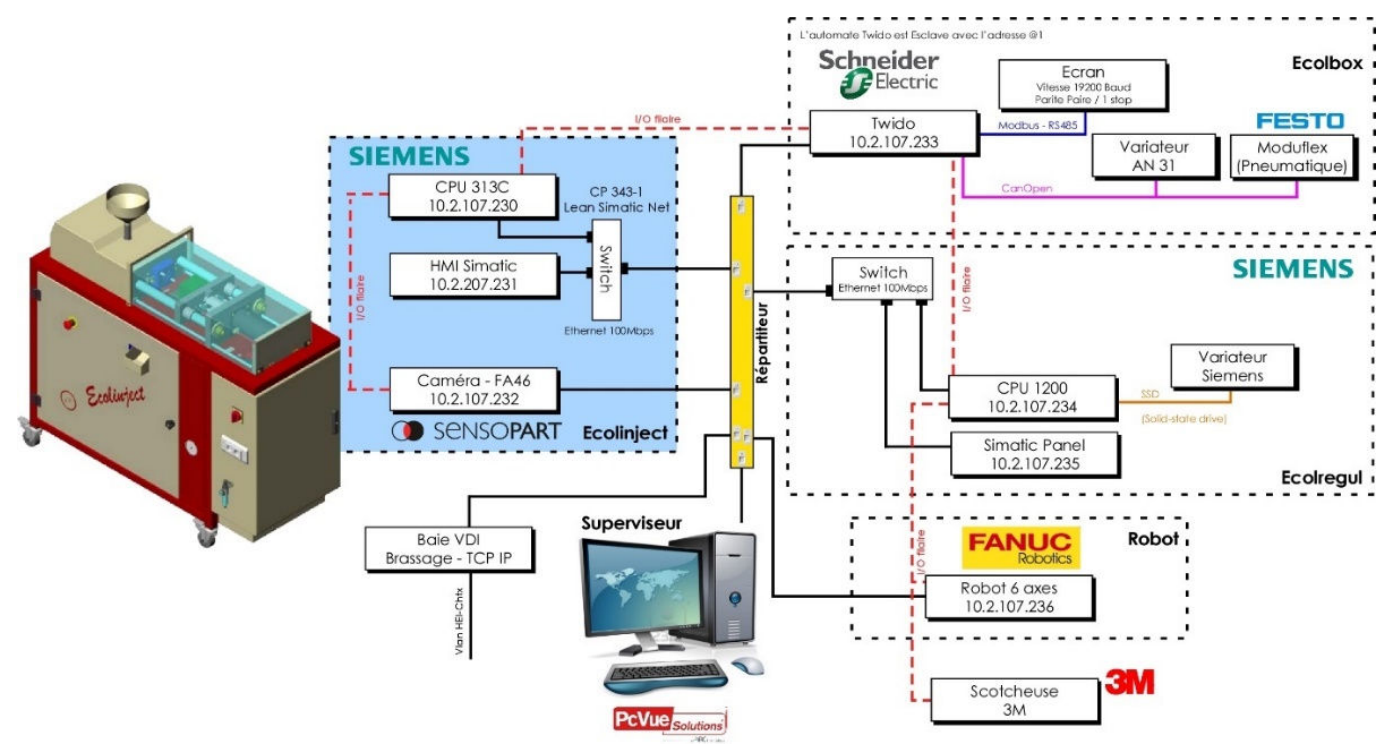

Figure 5. Architecture complète du réseau

Le challenge a concerné une partie de cette mini-usine : la partie Ecolinject. Cette partie intègre de nombreuses fonctions primaires que l'on retrouve sur un processus industriel : une fonction de régulation de température avec différentes recettes de fabrication, une fonction presse à injecter hydraulique, une fonction d'approvisionnement des billes en polymère vers un moule (4 empreintes), une fonction de contrôle de la qualité des jetons ainsi produits par l'intermédiaire d'une caméra (Figure 6). Le tout, est automatisé avec différents produits de la marque Siemens (Automate Programmable Industriel, pupitre opérateur, pré-actionneurs...).

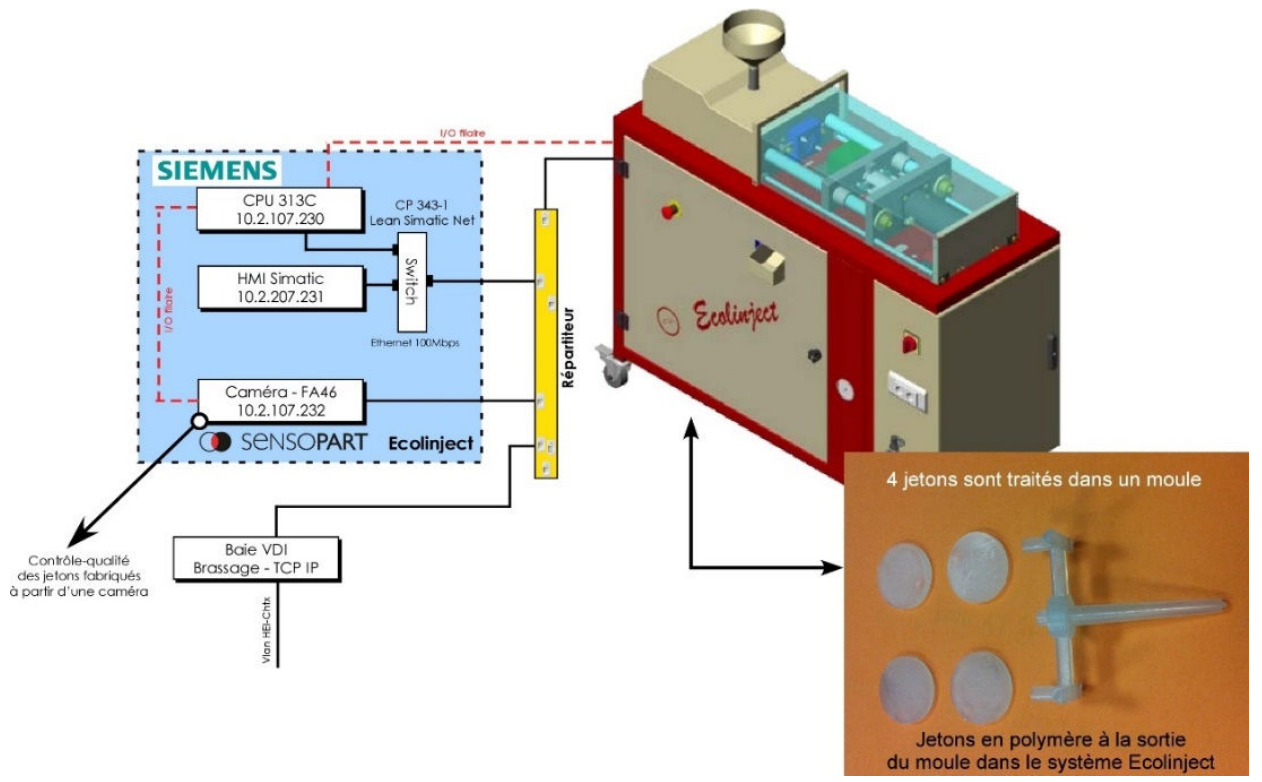

Figure 6. Détail sur 1'Ecolinject

Proposition d'article pour le Journal: J3eA, Journal sur l'enseignement des sciences et technologies de l'information et des systèmes 
La Vidéo 1 présente en détail l'ensemble fonctionnel pour la partie Ecolinject. Ce sera le cœur du support de travail pour le challenge.
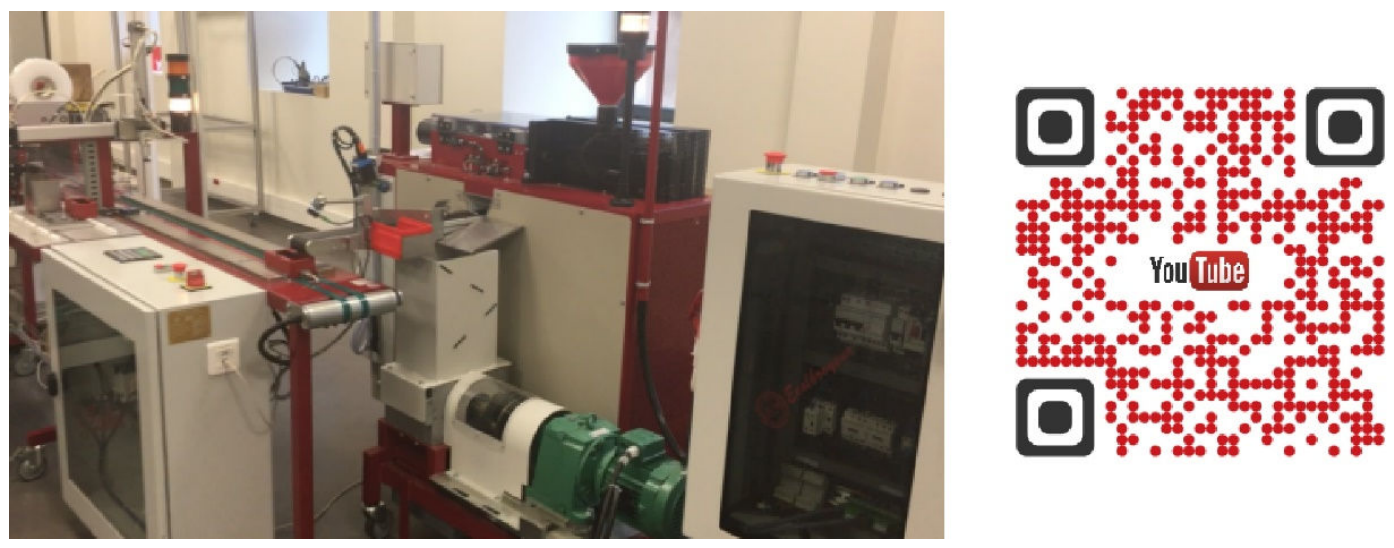

Vidéo 1 : Le système de fabrication de jetons - https://youtu.be/NCw1b8vUljQ

Le travail proposé a consisté à mettre en place une structure opérationnelle montrant les fondements d'une architecture industrielle communicante dans une démarche de monitoring de production. Il apporte un grand nombre de compléments d'informations par rapport aux articles [4], [20] et [23]. A partir des informations émanant du processus étudié, les différents groupes devaient développer et utiliser plusieurs clients dans une démarche SCADA ${ }^{1}$ [19]. Il fallait montrer dans ce travail plusieurs solutions à implémenter afin d'effectuer un large panel de tests. Pour cela, les données devaient être centralisées dans un serveur OPC $\mathrm{DA}^{2}$ et mises à disposition vers différents clients OPC (Figure 7). L'ensemble de la solution devait également permettre de suivre « en ligne » un certain nombre de contenus de variables (Items ${ }^{3}$ ) associés à la qualité des produits fabriqués. Les états de bon fonctionnement et de dysfonctionnement du système devaient également faire l'objet d'un travail tout particulier. Ce travail devait s'appuyer sur les travaux présentés dans [21] et [22]. Les informations sensibles liées à la production devaient être envoyées à un destinataire par le biais de deux stratégies d'émissions :

$\checkmark$ SMS avec un message associé sur un téléphone mobile (responsable de la maintenance ou de la production),

$\checkmark$ Mail avec un message associé sur une boite Mail (responsable de la maintenance ou de la production).

La Figure 7 présente en détails les différentes contraintes techniques que devaient gérer les élèves. Ce travail devait donc consister à venir capter des informations pertinentes sur le processus (information détecteur, information boutonnerie, information pré-actionneur, information caméra, information élément de sécurité...) sans perturber le fonctionnement du système de production. L'Automate Programmable Industriel à utiliser provenait du

\footnotetext{
${ }^{1}$ SCADA : Supervisory Control and Data Acquisition. L'objectif d'un environnement SCADA est de mener une conduite réactive d'un processus. Un environnement SCADA comprend 4 sous-ensembles : la fonction acquisition, la fonction commande, la fonction surveillance et la fonction supervision.

${ }^{2}$ La spécification d'accès aux données OPC est la première d'un groupe de spécifications connues sous le nom de spécifications OPC classiques. OPC Data Access est un groupe de normes client-serveur qui fournit des spécifications pour la communication de données en temps réel à partir de dispositifs d'acquisition de données tels que les Automates Programmables Industriels (API) vers des dispositifs d'affichage et d'interface comme les Interfaces Homme-Machine (IHM), les systèmes SCADA ainsi que les systèmes ERP/MES.

${ }^{3}$ Item : désignation d'un objet associé à une variable permettant d'être traitée par le serveur OPC et les clients potentiels.
} 
constructeur Phoenix Contact (ILC 151 GSM/GPRS). Une fois câblé, il devait permettre de traiter à la demande, les informations désirées par les différents clients (Figure 7). Un certain nombre de logiciels ont pour cela été installés sur les ordinateurs individuels des élèves. Les différentes licences et autorisations avaient été négociées au préalable avec les éditeurs. Ces plateformes logicielles étaient les suivantes: AutomationWorx 2018, Cogent DataHub V8, une suite bureautique (Office ou autre (> 2010)), Qelectrotech V0.7. Les solutions matérielles utilisées devaient s'insèrer bien évidement dans un contexte, où les communications numériques sans-fil étendent leur influence [5].

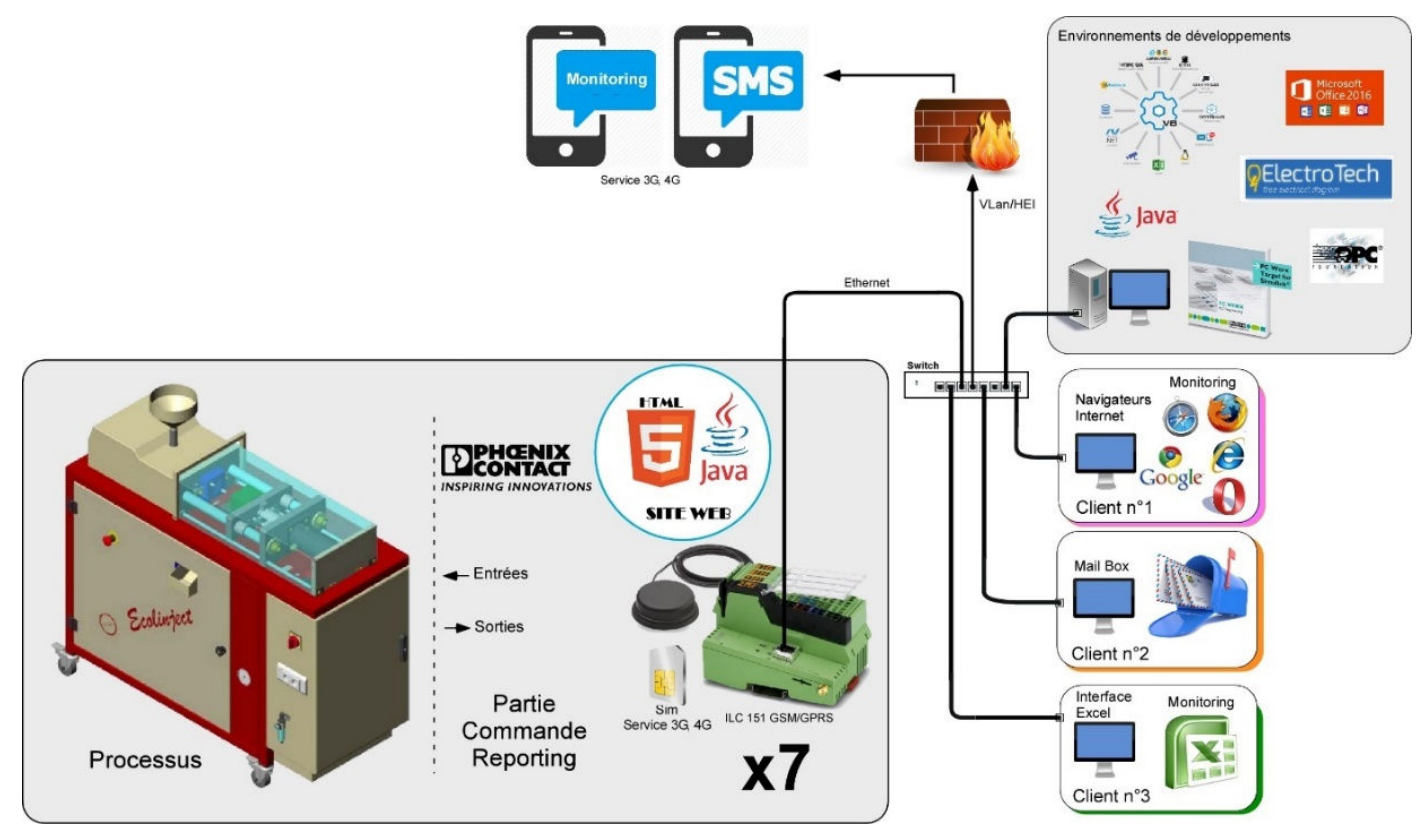

Figure 7. Les contraintes techniques du challenge

\subsection{Une collaboration avec un fabricant de solutions d'automatisation et un éditeur de logiciel}

Avec l'aide du réseau universitaire international EduNet ${ }^{4}$, Phoenix Contact encourage l'échange et la collaboration entre diverses Universités œuvrant avec le tissu professionnel dans le domaine de l'automatisation des processus ainsi qu'en Recherche et Développement. A ce jour, ce réseau international de contacts et de compétences regroupe 110 collèges (lycées) et Universités, le tout, dispersé dans 30 pays (Figure 8).

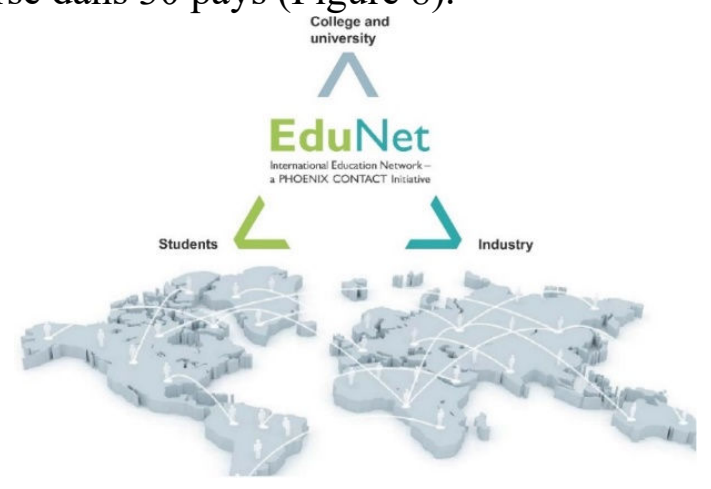

Figure 8. Le réseau Edunet

${ }^{4}$ https://elms.ccad.eu/moodle/

Proposition d'article pour le Journal: J3eA, Journal sur l'enseignement des sciences et technologies de l'information et des systèmes 
C'est dans ce cadre, que les structures de formations partenaires conçoivent leurs enseignements grâce au matériel Phoenix Contact offert par cette convention. En échange, elles doivent laisser à disposition de la communauté inscrite sur la plateforme EduNet leurs supports pédagogiques et expériences. De plus, des laboratoires «Phoenix Contact» sont initiés au sein d'Universités de manière collaborative facilitant ainsi, l'entrée des étudiants dans le monde professionnel. En juillet 2017, L'Université d'Orléans avec l'IUT de l'Indre signaient la première convention pour la France avec ce réseau [16]. Cette collaboration étroite depuis cette date nous a permis d'intégrer de nouvelles technologies pour le challenge avec des tarifs négociés d'achats de matériels et de logiciels (Figure 7). Une plateforme logicielle (PC Worx, Figure 9) mise à disposition permettra de configurer, de programmer et de diagnostiquer l'API (ILC 151 GSM/GPRS).

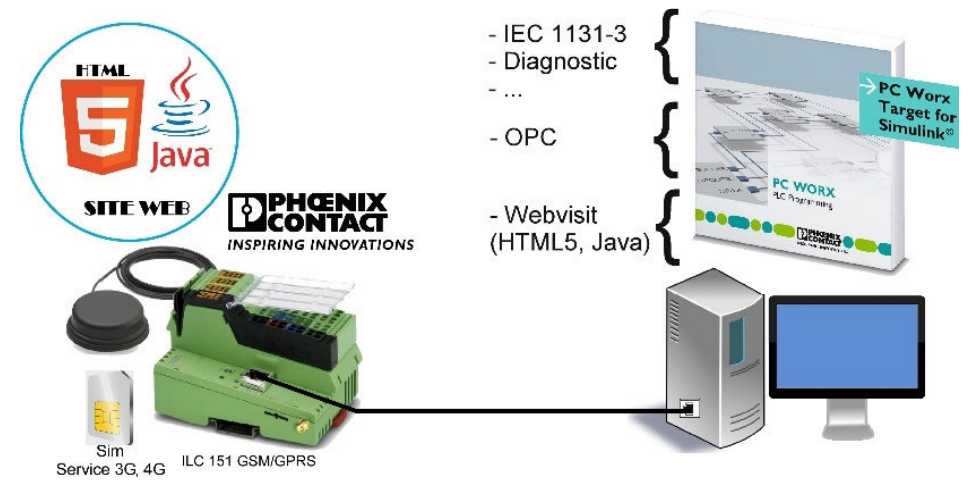

Figure 9. Plateforme de développement PC Worx

La $2^{\text {ème }}$ collaboration nous a permis d'intégrer un éditeur international de logiciels. Son nom est Skkynet [10]. Nous travaillons depuis 3 ans avec cet éditeur de logiciel et un environnement nommé Cogent DataHub. Cogent DataHub (Figure 10) est un outil unique qui permet de connecter, de concentrer, d'intégrer et de redistribuer des données en temps réel entre différents devices (API, ...) et utilisateurs (applications de supervision, base de données, ...).

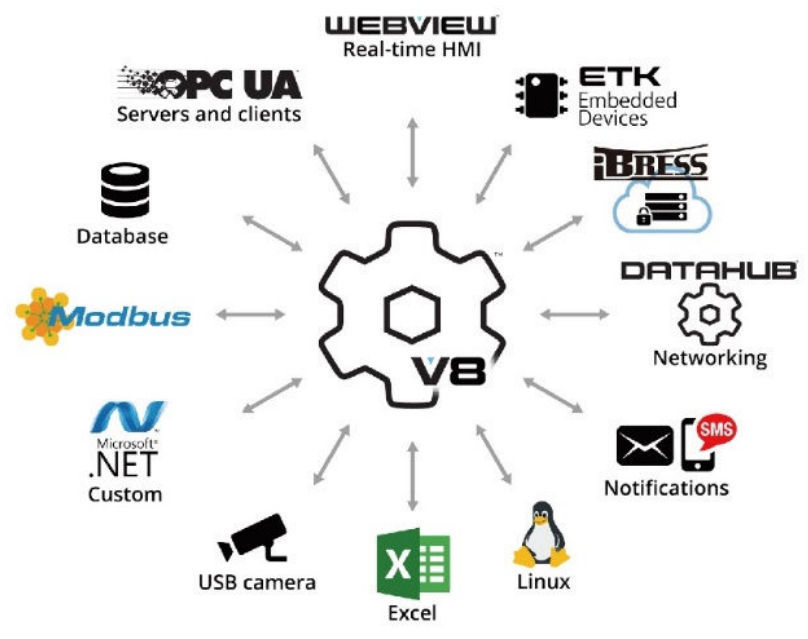

Figure 10. Cogent DataHub

L'éditeur a accepté de nous fournir les licences nécessaires pour le challenge afin que les élèves puissent utiliser cette plateforme sur leur ordinateur personnel. 


\section{La démarche pédagogique de travail adoptée pour le challenge}

Comme nous l'avons présenté dans l'introduction de cet article, nous avons adopté une démarche dite « pédagogie par projet ». Comme nous le présenterons par la suite, cette stratégie présente de nombreux intérêts avec des résultats obtenus très satisfaisants. Néanmoins, elle demande une préparation en amont très conséquente de la part de l'enseignant pour permettre de garantir des sorties de labyrinthe pour certains élèves. Tests unitaires préalables, planning prévisionnel et critères d'évaluation doivent être correctement validés et dimensionnés. L'expérience des années dans ce métier de formateur apportera de nombreuses solutions.

Le challenge proposé a été soumis à des élèves ingénieurs apprentis en 5 ème année. Ces élèves sont en formations mécatronique et robotique et ne sont donc pas des spécialistes en informatique industrielle et réseaux de communications numériques. Le groupe était constitué de 30 personnes. Les diplômes antérieurs de ces élèves étaient : DUT 53\%, BTS 27\%, Licence ou Classe Préparatoire 20\%. Avant le démarrage du challenge, un tirage au sort a été effectué avec les participants afin de ne pas créer a priori, ni d'affinité préalable ni de compétences déséquilibrées au sein d'un groupe. Sept groupes ont ainsi été constitués le 16 octobre 2018 (Figure 11). Chaque groupe devait choisir un(e) leader (médiateur, organisateur, formateur, rapporteur...). Le sujet a été présenté avec un formalisme de type $\mathrm{STB}^{5}$ associé aux différents critères d'évaluations attendus. La mise à disposition de l'ensemble des ressources documentaires a également été effectuée lors de la $1^{\text {ère }}$ séance de travail (Figure 11).

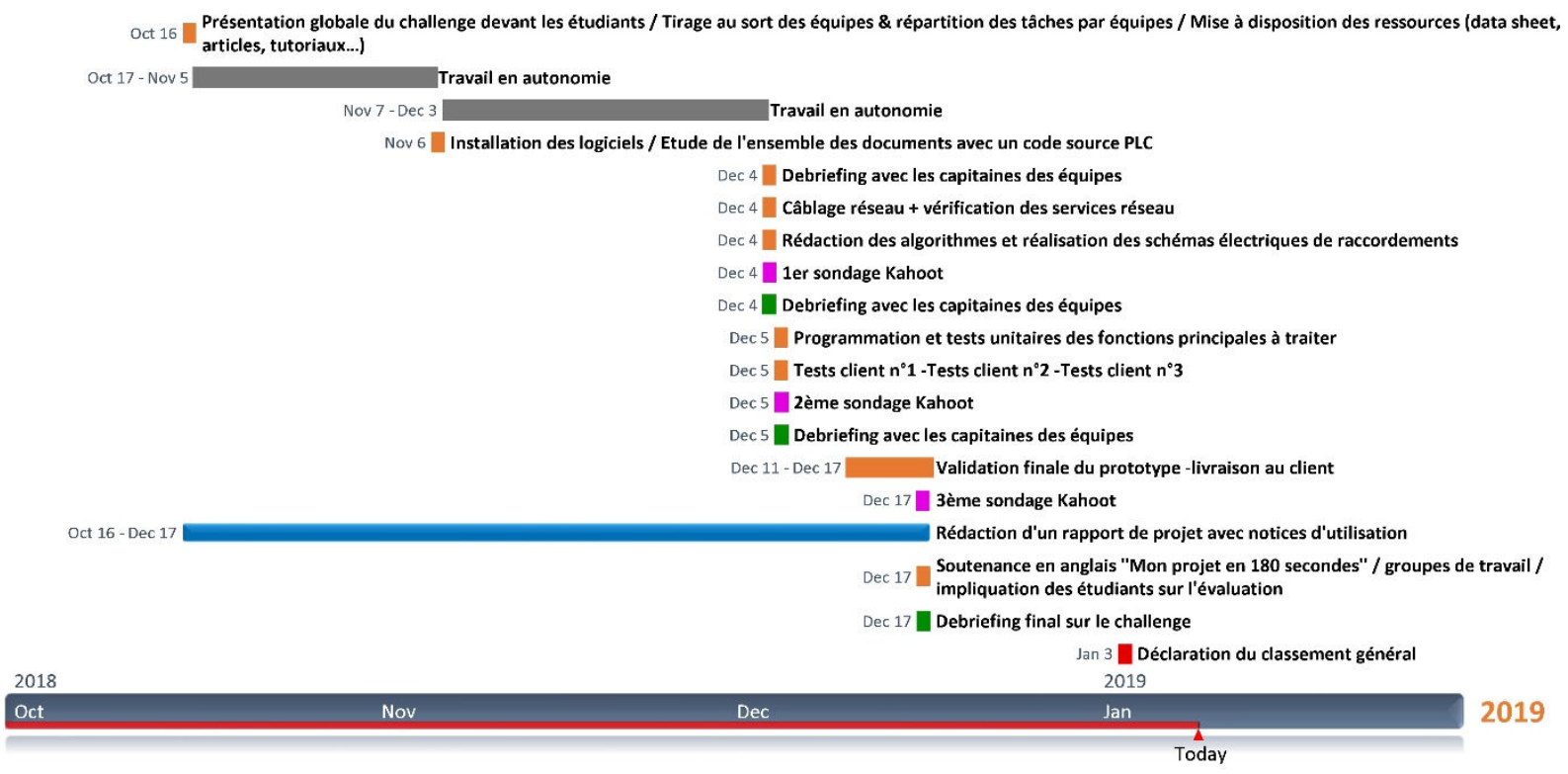

Figure 11. Le planning prévisionnel de travail

Le challenge a fait l'objet d'un travail encadré de 35 heures. Deux périodes ont été consacrées spécifiquement au travail en autonomie. Un Fablab était mis à disposition avec une possible utilisation tard le soir $(02 \mathrm{~h} 00)$ ou sur la pause déjeuner. Son accès sécurisé est géré avec un lecteur de badges.

\footnotetext{
${ }^{5}$ STB : La Spécification Technique de Besoin est un document qui traduit le besoin d'un demandeur en termes d'exigences et de contraintes techniques (spécifications). La STB doit être suffisante pour qu'un concepteur puisse élaborer une définition du produit afin d'y répondre sans ambiguité.
} 
Le planning prévisionnel proposé dès le début du challenge (Figure 11) fait également apparaitre des séances de «Debriefing » avec les leaders d'équipe et des séances de sondages placées au fil de l'eau. Ces dernières ont eu plusieurs vocations :

$\checkmark$ Insérer officiellement l'utilisation du Smart-Phone personnel de chaque élève dans la formation (ce dispositif présent dans la poche de chaque élève devient un moyen technologique au service de la pédagogique),

$\checkmark$ Apporter une dynamique pédagogique «moderne» dans le cadre de sondages ou de QCM,

$\checkmark$ Disposer d'un outil disponible sur internet afin de pouvoir tenter de caractériser la représentation adaptée de la dynamique de la situation dite « projet» (Figure 2).

La séance de « Debriefing » avait plusieurs objectifs pédagogiques :

$\checkmark$ Vérifier l'organisation au sein des groupes (Qui fait quoi par rapport aux tâches internes fixées préalablement par le groupe ?),

$\checkmark$ Vérifier l'état d'avancement du challenge et anticiper les actions à venir,

$\checkmark$ Répondre à des besoins techniques en ouvrant certains verrous,

$\checkmark \quad \ldots$

Une stratégie complémentaire pédagogique a également été mise en œuvre à base d'une pédagogie dite «active» [1]. Cette stratégie avait pour vocation de valider la capacité de restitutions d'informations de la part de certains élèves vers d'autres élèves (leader vers autres participants du groupe). Compte tenu des nombreuses tâches à traiter, cette stratégie a permis également de ne pas mobiliser toutes les ressources humaines au même moment avec l'enseignant. Les apports de connaissances spécifiques sur les différents environnements logiciels ont été diffusés uniquement aux leaders (Figure 12 (a)). Les leaders devaient ensuite restituer en fonction des besoins, les connaissances qu'ils avaient acquises préalablement (Figure $12(b)$ ).
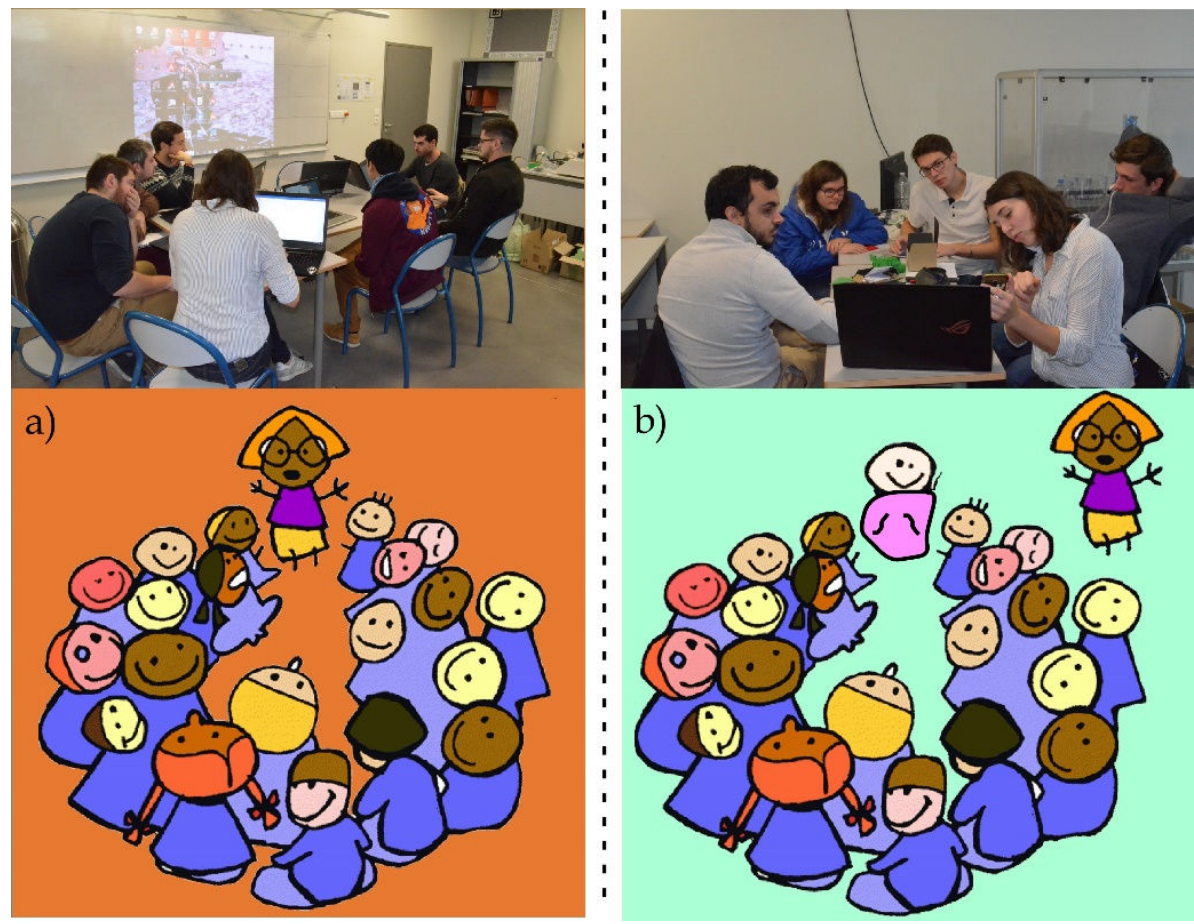

Figure 12. Principe de la stratégie adoptée en matière de pédagogie inversée

Proposition d'article pour le Journal: J3eA, Journal sur l'enseignement des sciences et technologies de l'information et des systèmes 


\section{Des résultats significatifs obtenus par différents groupes}

Très souvent, face à un problème posé et à résoudre, notre processus de raisonnement nous propose trop rapidement la ou les solutions avant de contextualiser intellectuellement le sujet ou l'exercice à traiter. Ce réflexe est souvent présent pour nous rassurer, subterfuge intellectuel pour ne pas sortir de notre cercle de confiance [12]. Nous avons tous connu cet état de fait. Parfois, l'exercice à traiter prête à une réaction et une action très rapide. Il faut réagir au mieux dans l'instant... Néanmoins, et très souvent, de nombreux problèmes peuvent et doivent être contextualisés avec l'usage d'outils de formalisation bien répertoriés dans la littérature [6], [7]. Avant toutes interventions opérationnelles, les différents groupes devaient se concerter afin de faire ressortir les informations indispensables émises par le système (sans modification ni du programme stocké dans l'API et le pupitre opérateur, ni du câblage de l'armoire électrique). La majorité des groupes a souhaité pouvoir traiter les informations suivantes :

$\checkmark$ Gestion des modes de marche et d'arrêt: système en marche ou arrêté, arrêt d'urgence, disjonction de la protection électrique de la bande transporteuse, ouverture-fermeture capot de protection, bourrage, dégrappage, protection thermique des colliers de chauffe présents avant le moule.

$\checkmark$ Gestion de la recette de fabrication : ouverture et fermeture du moule, position du vérin hydraulique, information sur la qualité des jetons, quantité de jetons produits.

Pour mener à bien ce sujet, les Figure 13 et 14 décrivent le travail de concaténation qui a été effectué dans une concertation globale entre les différents groupes. Les documents techniques du constructeur du système ont dû être étudiés avec le plus grand sérieux.

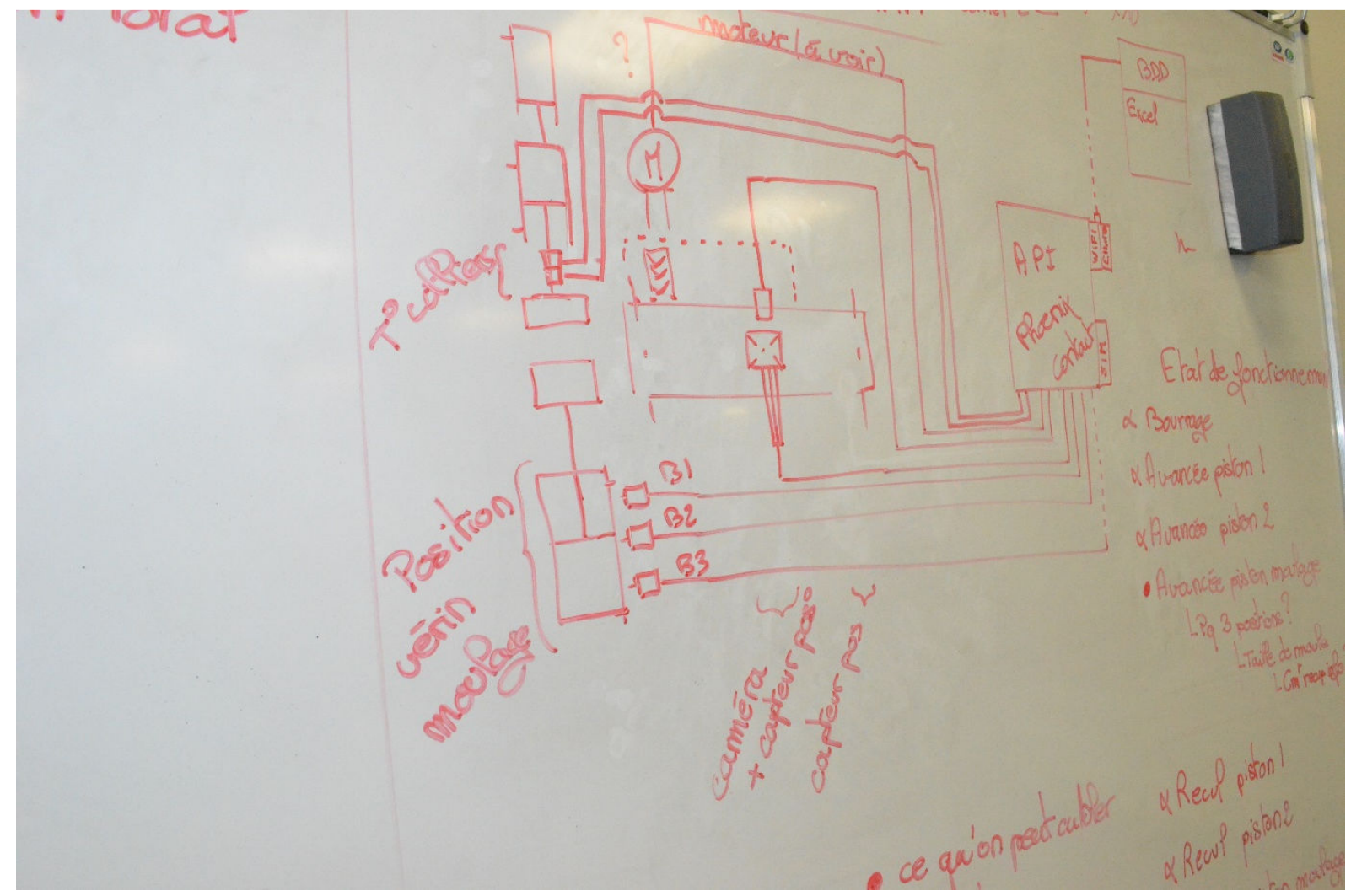

Figure 13. Travail de concaténation des informations à traiter 


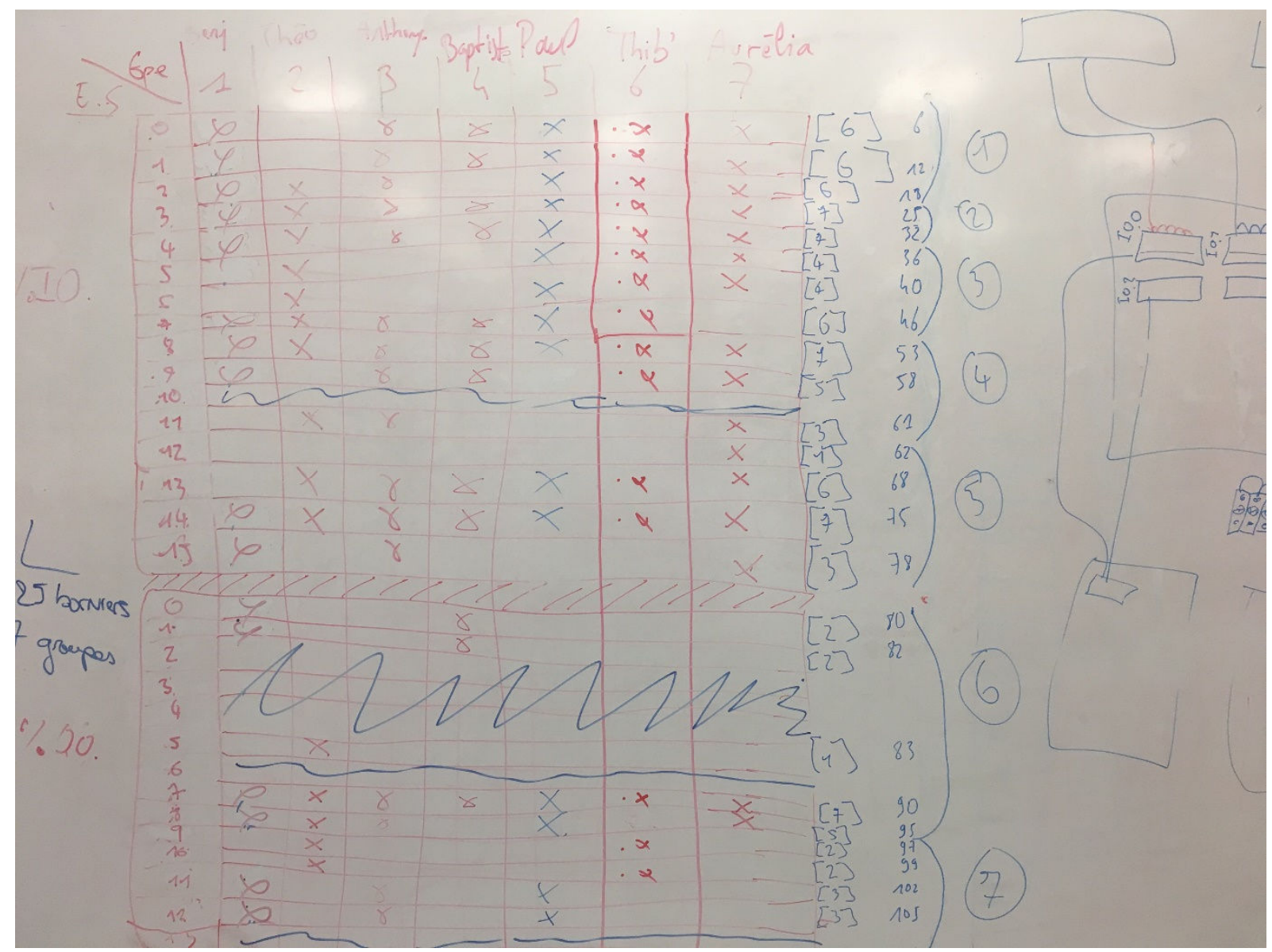

Figure 14. Travail de concaténation des informations à traiter

La principale contrainte électrique (Figure 15) consistait à permettre l'accessibilité des différentes informations à l'ensemble des groupes, tout en respectant les éléments exprimés (Figure 13). L'alimentation générale (24VDC) disponible dans l'armoire électrique permettait un raccordement sur l'ensemble des 7 platines (Figure 15).

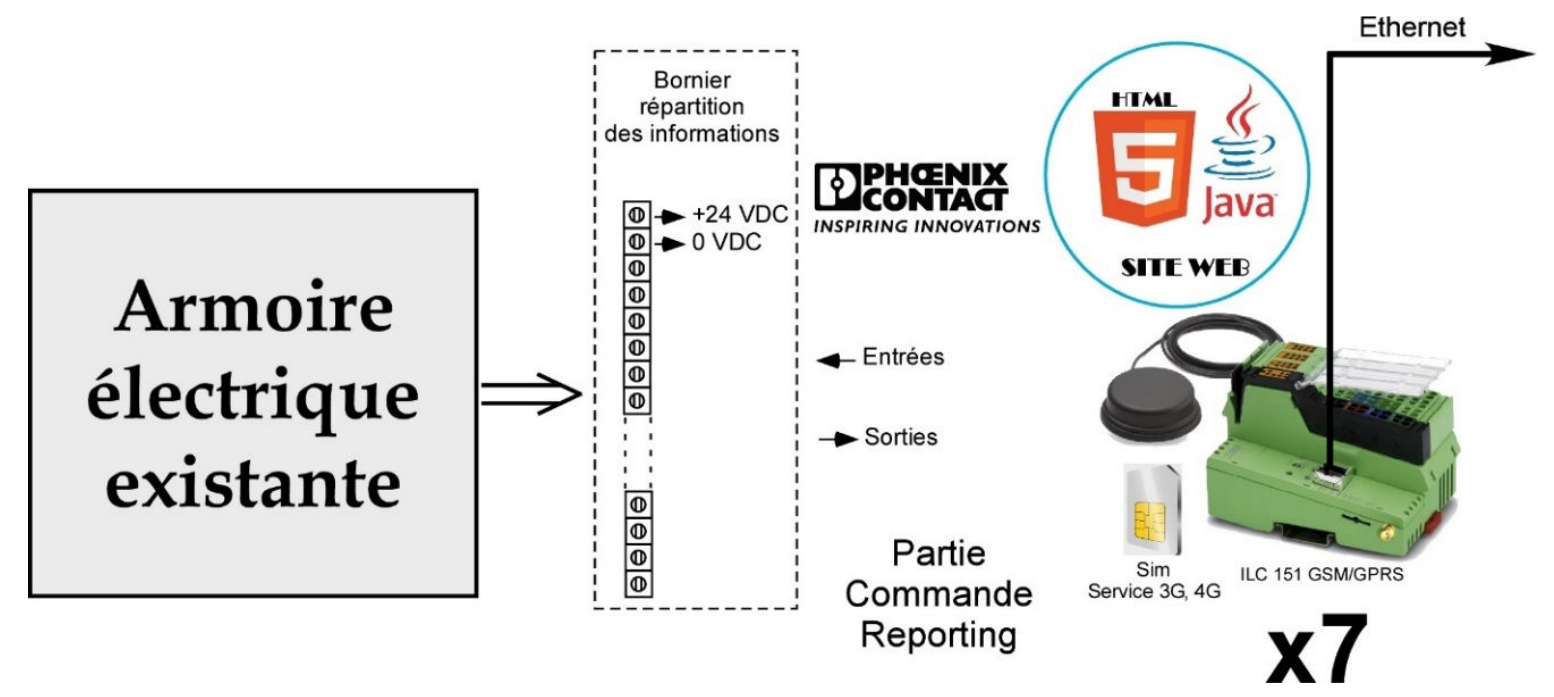

Figure 15. Contraintes de raccordements électriques

La Figure 16 présente le travail de câblage qui a été nécessaire pour mettre à disposition l'ensemble des signaux nécessaires et préalablement choisis par les différentes équipes.

Proposition d'article pour le Journal: J3eA, Journal sur l'enseignement des sciences et technologies de l'information et des systèmes 

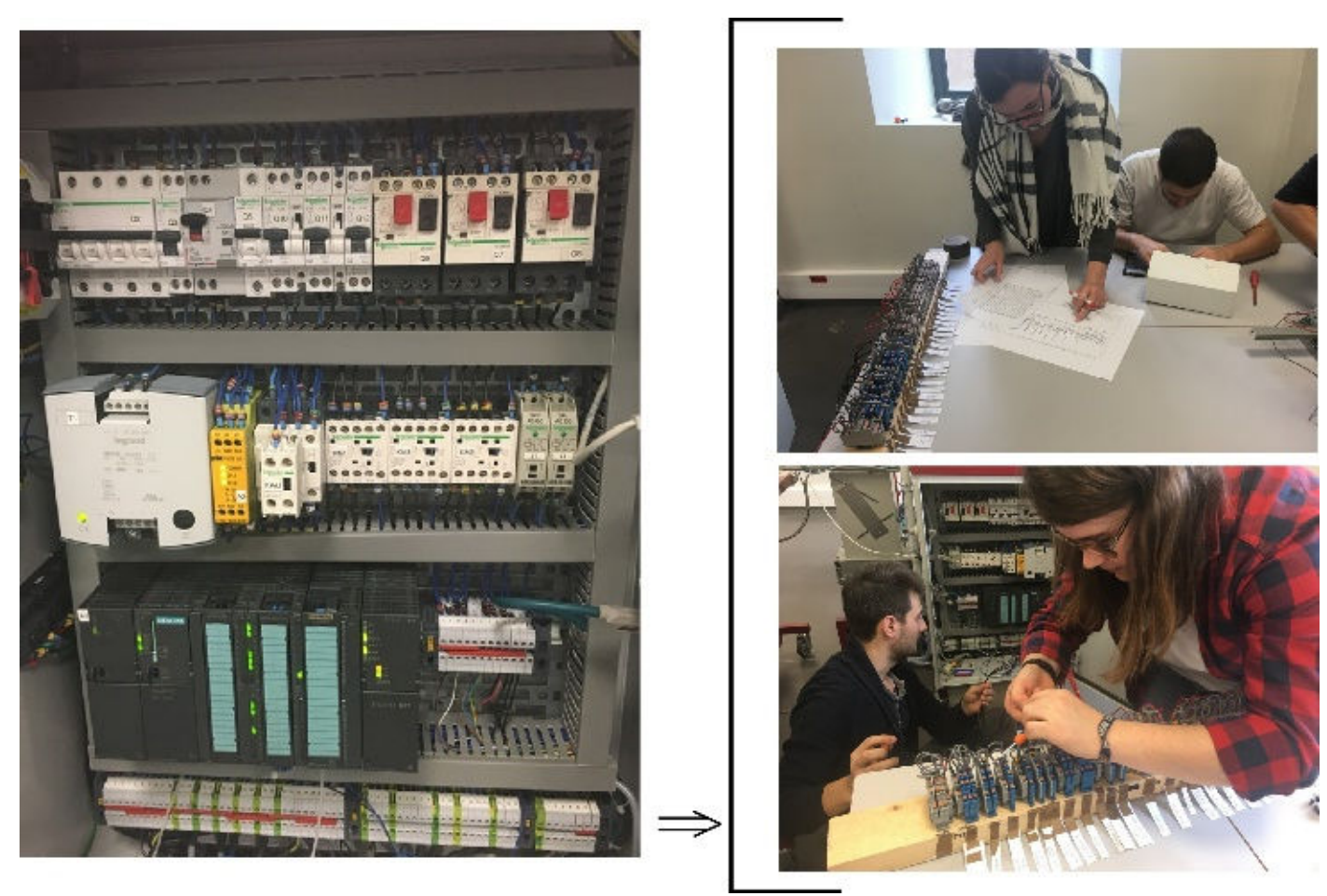

Figure 16. Contraintes de raccordements électriques

Comme nous l'avons précisé, chaque groupe disposait de l'ensemble des ressources pour mener à bien le challenge. La Figure 17 présente une platine en cours de montage : (a), l'API et sa carte SIM (abonnement à choisir chez un fournisseur d'accès en téléphonie) ; (b), un début de câblage et de programmation pour un groupe.

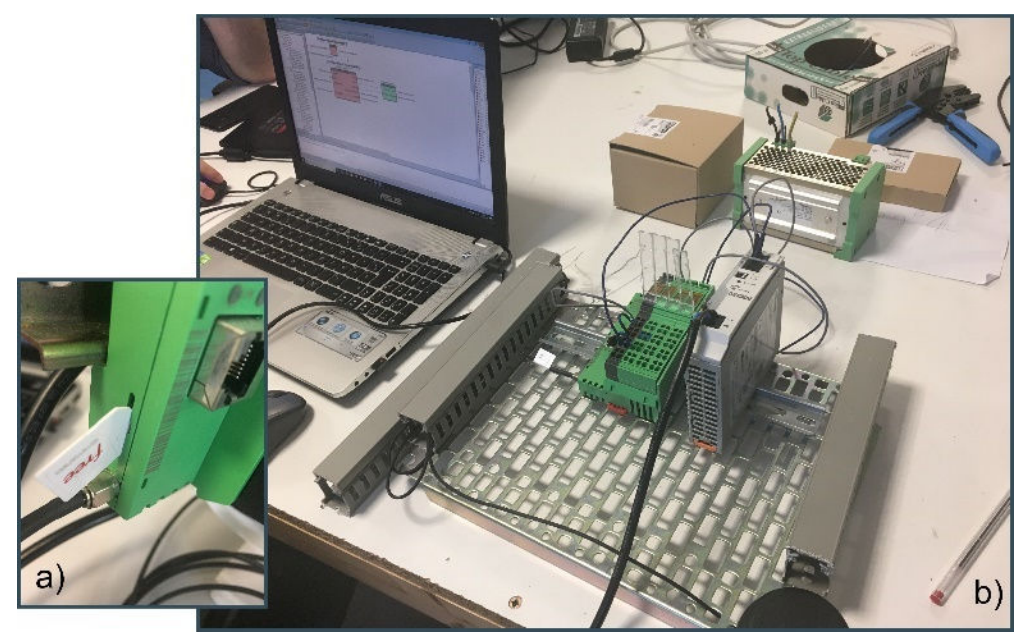

Figure 17. Phase intermédiaire de développement

La Figure 18 (a) présente le résultat final concernant l'ensemble des interconnexions nécessaires. Les élèves ont été particulièrement sensibilisés aux contraintes pouvant être liées à un câblage électrique. Chaque groupe a également géré l'obligation de vérifications des premières conformités que l'on doit assurer avant le démarrage d'une telle installation (Figure 18 (b)). Ces conformités concernent les potentiels électriques, les sécurités électriques, les états des entrées (détecteurs, capteurs), l'état initiale de la partie opérative, l'état initial du programme dans l'API... 


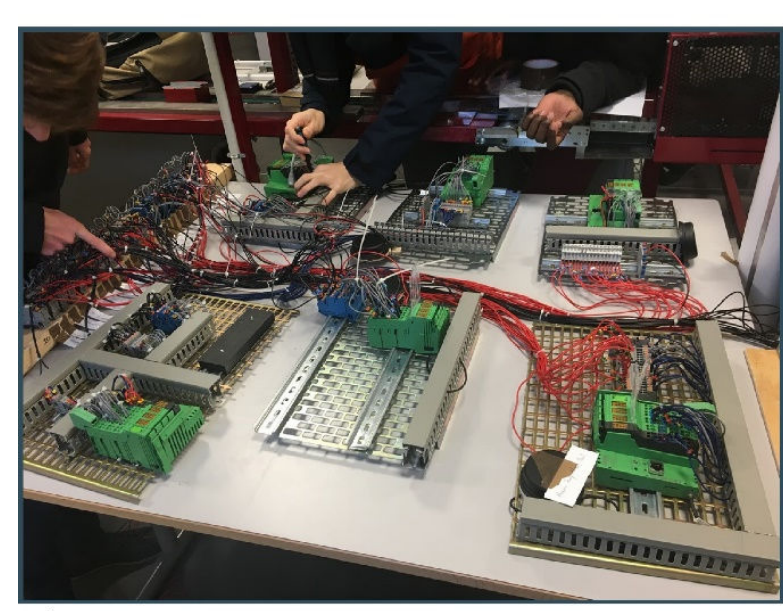

a)

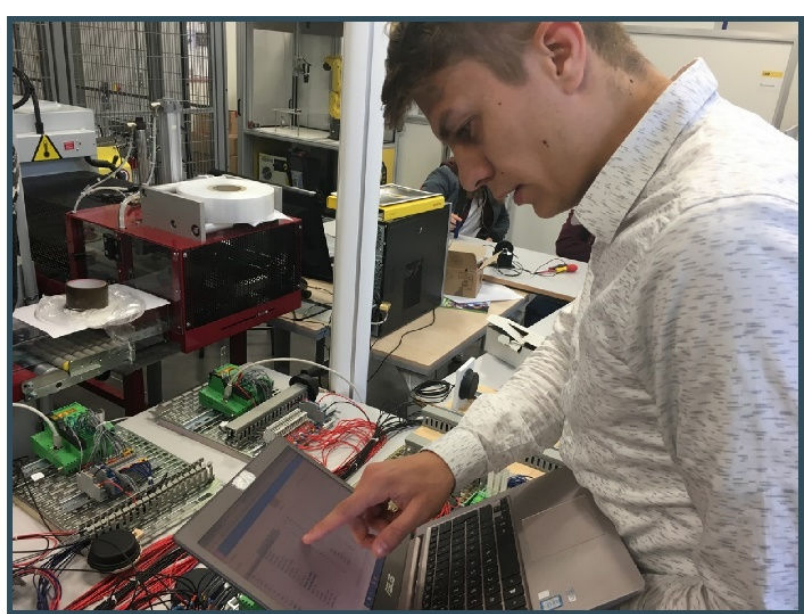

b)

Figure 18. Interconnexions globales et phase de tests

Les résultats obtenus ont été de très bonne qualité. Le résultat final pour un groupe est présenté dans la Vidéo 2. La vidéo devait être réalisée avec une seule et unique prise de vue incluant les différentes démonstrations. Les élèves pouvaient réaliser au préalable, autant d'essais qu'ils le souhaitaient.

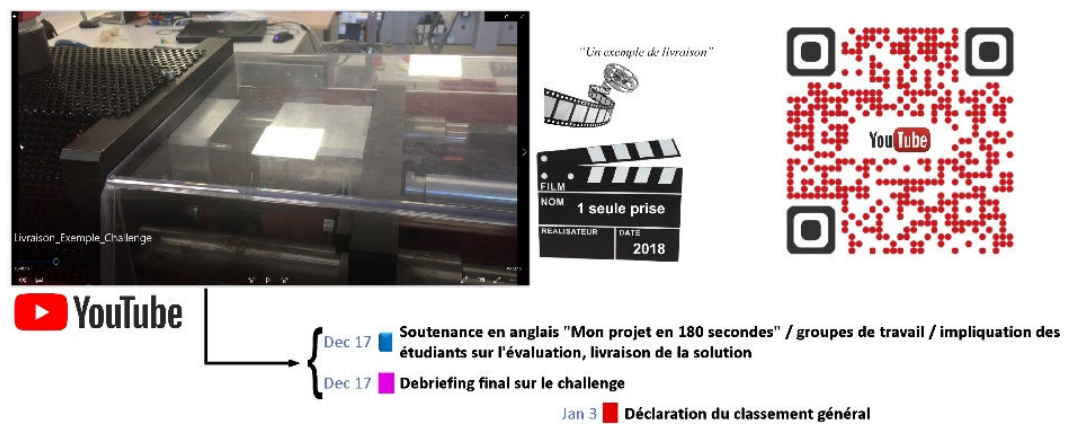

Vidéo 2 : Un exemple de résultats - https://youtu.be/RTw6IUp57JY

\section{Benchmark pédagogique}

Le premier retour sur expérience très positif qui ressort de ce challenge concerne les résultats techniques obtenus par les 7 groupes. Non spécialistes du domaine et découvrant lors de ce challenge l'ensemble des matériels et des logiciels, les élèves ont été particulièrement performants et organisés. La Figure 11 montre trois sondages effectués avec Kahoot. Dans notre cas, il a été utilisé pour :

\footnotetext{
$\checkmark$ Redynamiser les groupes en phase de redémarrage... ou d'endormissement !

$\checkmark$ Établir un diagnostic sur le travail des élèves et leur motivation,

$\checkmark$ Évaluer le challenge,

$\checkmark$ Évaluer son propre travail (enseignant).
}

La Figure 19 présente un exemple de questions auxquelles devaient répondre les élèves. Une séance de sondage durait environ 5 minutes (temps de mise en place, temps pour les réponses). Les principaux résultats ont été présentés aux élèves lors de la séance de debriefing final sur le challenge. 


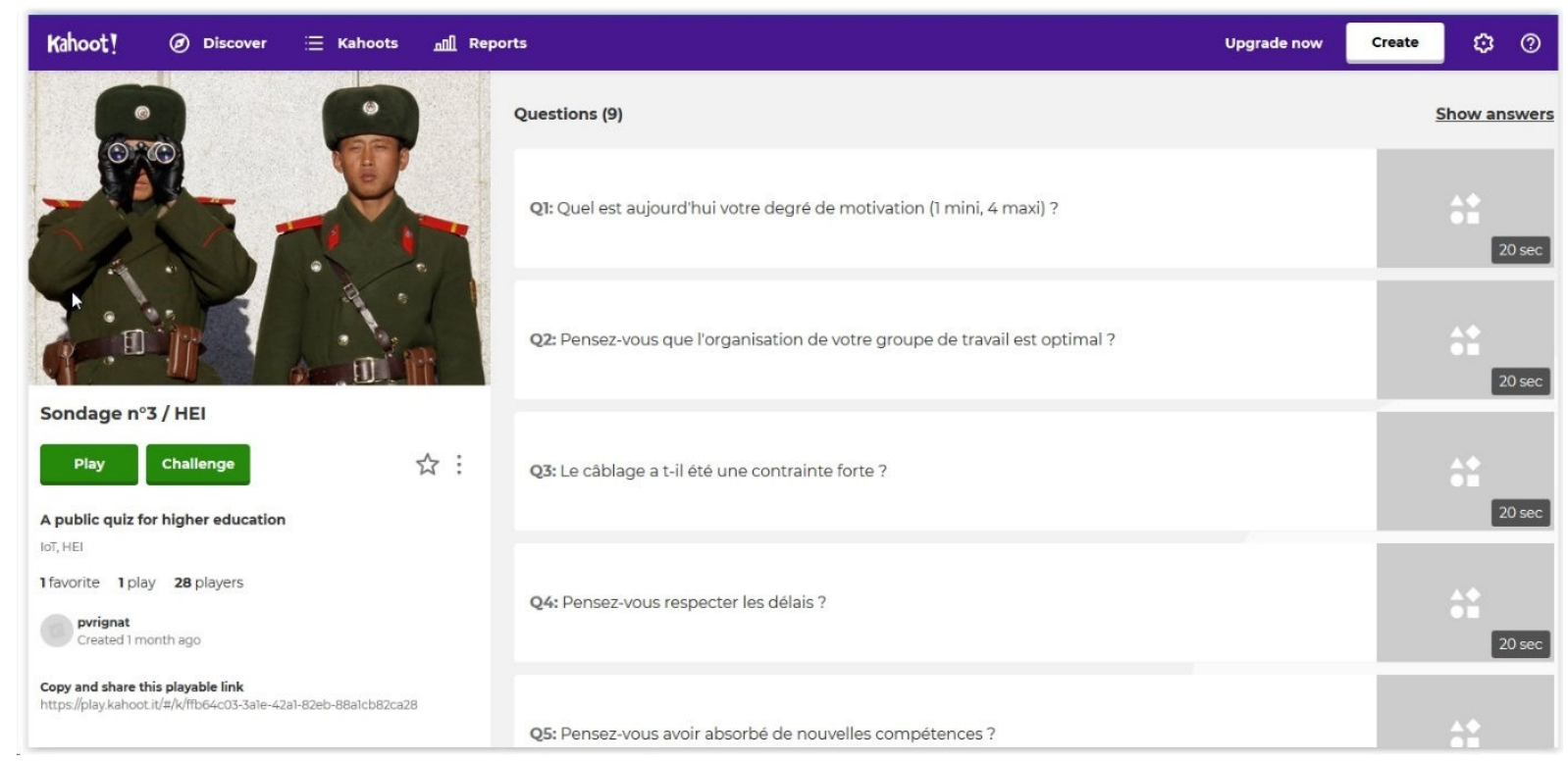

Figure 19. Un exemple de questions pour le Kahoot n³

Les résultats établis par l'outil sont présentés dans la Figure 20. Nous pouvons constater effectivement une augmentation du degré de motivation entre le début et la fin du challenge. Ce degré était à 2.8 au début et termine à 3.6 (sur 4 (note maximum)). Également interrogé sur leur niveau individuel de connaissances préalables, les résultats ainsi obtenus sont significatifs (1.14 au début du challenge et 3.8 à la fin). Les élèves ont largement augmenté leurs connaissances et compétences après le challenge. Nous montrons également que le degré de capacité d'action sur le challenge a été très important au début pour décliner au fur et à mesure de l'état d'avancement du travail.

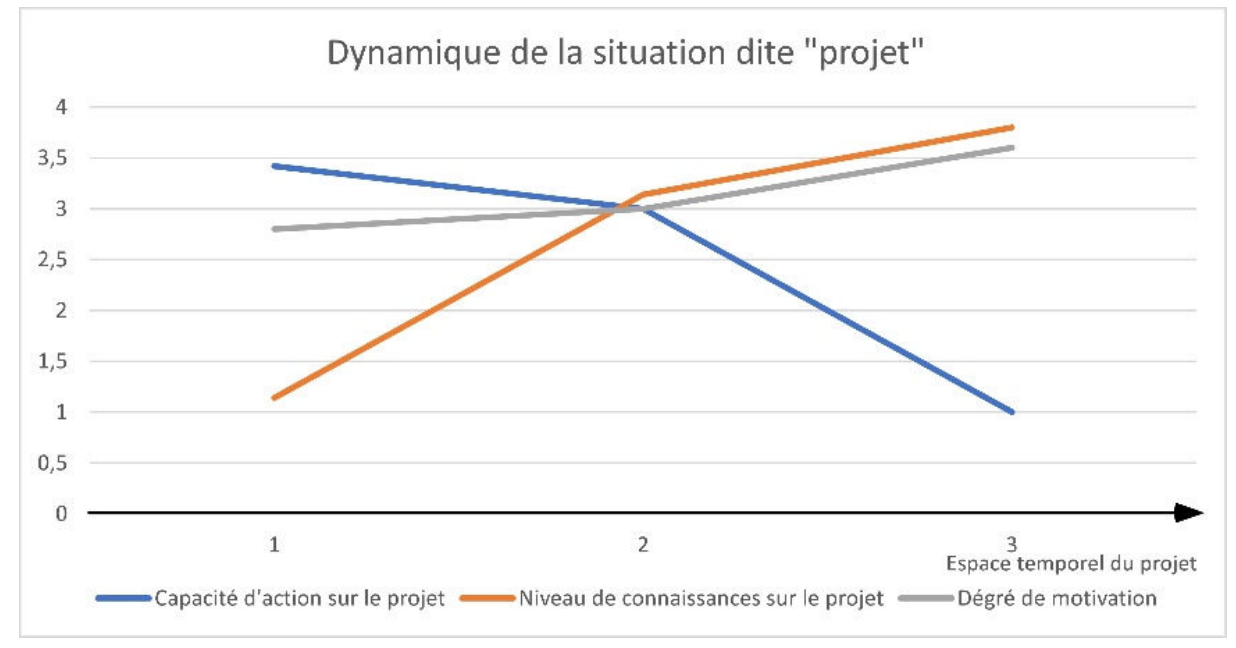

Figure 20. Résultats des sondages avec Kahoot

Le planning présenté dans la Figure 11 fait apparaitre une présentation en anglais pour chaque groupe de travail. C'était une implication forte également demandée avec un timing à respecter (présenter votre solution en $180 \mathrm{sec}$ avec une prise de vidéo unique). Lors des questions soumises avec Kahoot, une portait sur : « Le rendu oral en anglais $(180 \mathrm{sec})$ est-il une contrainte forte pour vous ? ». 85.7\% des élèves ont répondu par non. Ce résultat également significatif, montre le degré d'acceptation et la motivation des élèves sur ce sujet. Il faudra néanmoins travailler ce sujet pour $14.3 \%$ d'entre eux qui ne semblent pas encore très motivés par ce 
contexte alors qu'il est fondamental. Un résultat est présenté dans la Vidéo 3. Pour les présentations en anglais, l'ensemble des participants (élèves et enseignant) disposaient d'une feuille d'évaluation préalablement distribuée et présentée. La note attribuée pour la présentation de chaque groupe était une moyenne calculée entre toutes les feuilles. On pourra retenir sur ce sujet, que les élèves ont tendance à être plus « exigeants » sur l'évaluation que l'enseignant.

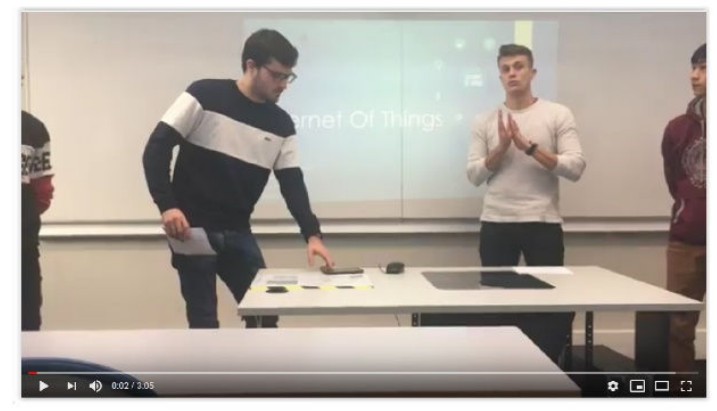

\section{YouTube}
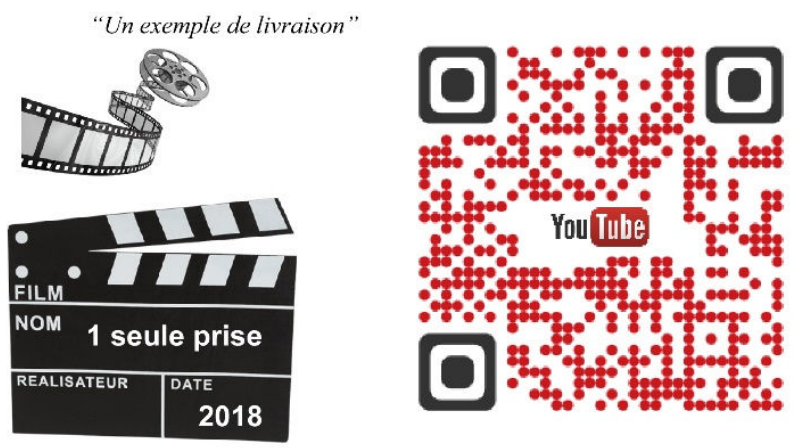

Vidéo 3 : Un exemple de résultats - https://youtu.be/9gwX7VarkfM

\section{Conclusion}

Les écoles d'ingénieurs sont adeptes des challenges depuis de nombreuses années (challenges internes, inter-écoles, nationaux voire internationaux). Ce challenge initié pour la

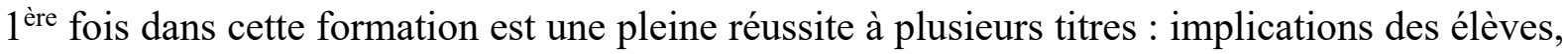
implications des professionnels externes, résultats obtenus, motivation des élèves, accroissement important de compétences individuelles et collectives... Nous avons montré que les pédagogies dites «par projet» et «actives" peuvent être une très bonne stratégie d'enseignement. Mais comme nous l'avons montré, de nombreux travaux préalables doivent être menés de la part de l'enseignant. Une remise des prix pour les 3 premières équipes a eu lieu au mois de janvier 2019.

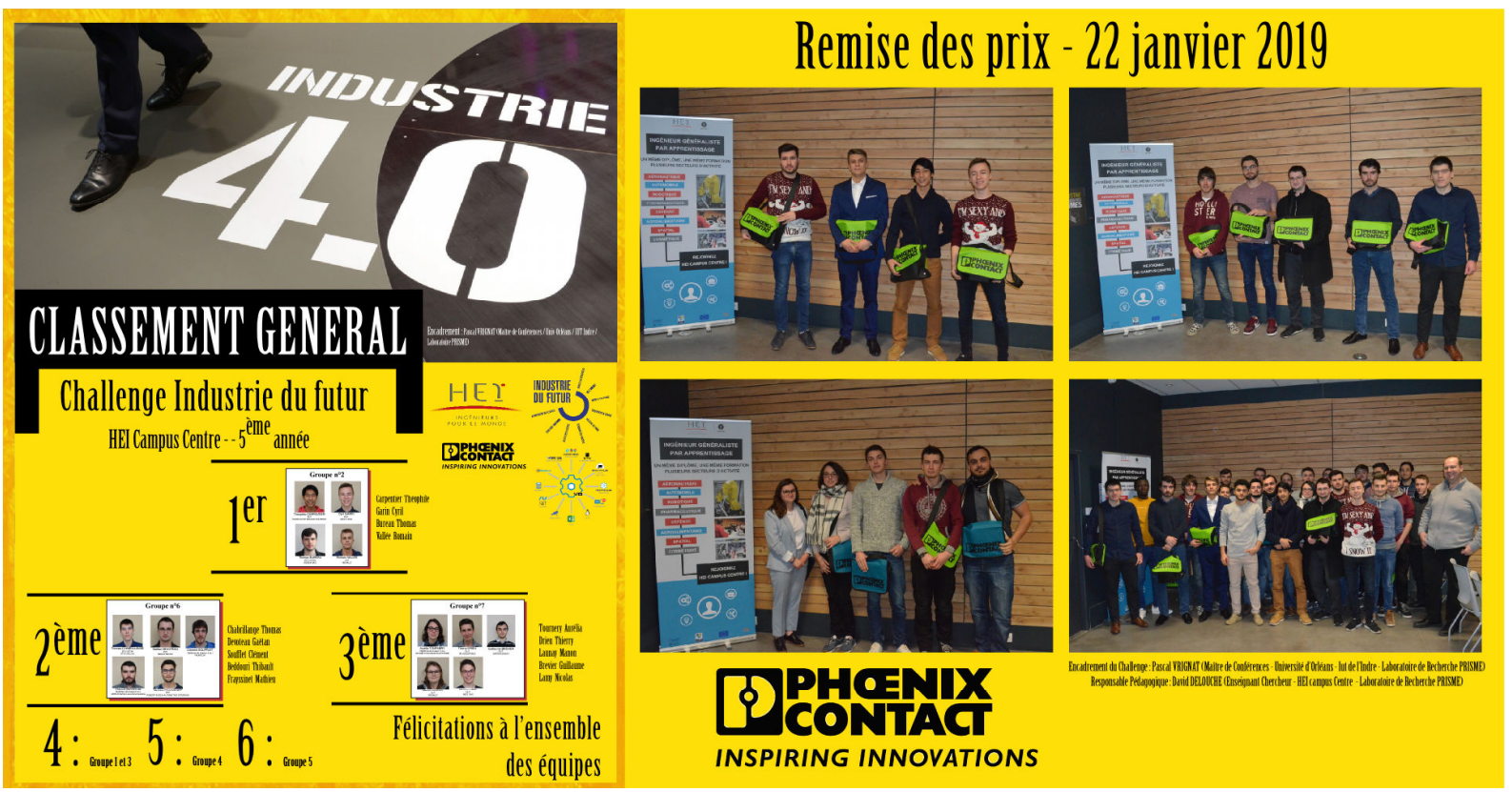

Figure 21. Remise des prix

Proposition d'article pour le Journal: J3eA, Journal sur l'enseignement des sciences et technologies de l'information et des systèmes 


\section{Références bibliographiques}

[1] S. Amégan, Pour une pédagogie active et créative, PUQ, 1993.

[2] S. Begot, F. Duculty, M. Avila, P. Vrignat, J.F. Millet and J.C. Bardet, Une possible réponse ludique pour les processus industriels communicants, 9ème Colloque sur l'Enseignement des Technologies et des Sciences de l'Information et des Systèmes (CETSIS 2011), 2011.

[3] P.C. Blumenfeld, E. Soloway, R.W. Marx, J.S. Krajcik, M. Guzdial and A. Palincsar, Motivating project-based learning: Sustaining the doing, supporting the learning, Educational psychologist, 26 (1991), pp. 369-398.

[4] J.P. Chemla and B. Riera, Programmation objet pour une domotique réalisée avec un API, Actes du 10ème Colloque sur l'Enseignement des Technologies et des Sciences de l'Information et des Systèmes (CESTIS-EEA'13), Caen, 2013, pp. 78-80.

[5] Communication Radio, Le sans-fil étend son influence, Mesures 843, 843 (2012), pp. 22-26.

[6] P.H. Des Mesnards, Réussir l'analyse des besoins, Editions Eyrolles, 2011.

[7] G. Garel, Pour une histoire de la gestion de projet, Gérer et comprendre, 74 (2003), pp. 77-89.

[8] HTTP://WWW.BEMA.FR/.

[9] $\quad$ HTTPS://KAHOOT.COM/.

[10] HTTPS://SKKYNET.COM/.

[11] L. Champaney, Pas d'industrie du Futur sans coopération entre industriels et enseignement supérieur, La Tribune (2017).

[12] S. Lenfle and C. Midler, Management de projet et innovation, Encyclopédie de l'Innovation (2003), pp. 49-69.

[13] C. Midler, L'auto qui n'existait pas, Management des projets et transformation de (1993).

[14] O. Lluansi and A. Galloni, Croire en l'Industrie du futur et au futur de l'industrie, Ernst \& Young Advisory (2017), pp. 1-45.

[15] P. Darmayan, Révolution humaine? Un nouveau rôle pour les hommes et les femmes de l'industrie du futur, Livre blanc de l'Agora Industrie (2018), pp. 1-56.

[16] PHOENIX CONTACT, Update 2|17, Le magazine client de Phoenix Contact (2017), pp. 1-28.

[17] J. Proulx, L'apprentissage par projet, PUQ, 2004.

[18] C. Reverdy, Des projets pour mieux apprendre?, Institut Française de l'Education, 82 (2013), pp. 1-24.

Proposition d'article pour le Journal: J3eA, Journal sur l'enseignement des sciences et technologies de l'information et des systèmes 
[19] B. Riera, F. Gellot, O. Dubois, J. Chemal and S. Triki, L'utilisation pédagogique et l'enseignement des TIC dans les automatismes, J3eA, 4 (2005), pp. 013.

[20] P. Vrignat, M. Avila, F. Duculty, S. Begot and J.F. Millet, Utilisation d'une architecture logicielle dans le cadre d'une mise en place d'une solution multi-clients via un serveur OPC, Gesi $n^{\circ} 77$, Revue des Départements : Génie Electrique et Informatique Industrielle en IUT, 2012.

[21] P. Vrignat, M. Avila, J.F. Millet, B. Roblès, F. Duculty, S. Begot, C. Bardet, D. Delouche, T. Aggab, J. Thuillier and F. Kratz, Des exemples de briques technologiques dans le cadre d'une application pour l'industrie du futur, 3EI (2018).

[22] P. Vrignat, F. Duculty and S. Limousin, Suivi d'une campagne d'irrigation par un enrouleur d'arrosage via un SmartPhone, La Revue 3E. I (SEE) (2017), pp. 41-50.

[23] P. Vrignat, J.F. Millet, F. Duculty, S. Begot and M. Avila, Développement et utilisation d'applications complémentaires dans une approche SCADA: retour sur expérience avec des étudiants $\mathrm{Bac}+2$ et $\mathrm{Bac}+5$, CETSIS, Colloque sur 1'Enseignement des Technologies et des Sciences de l'Information et des Systèmes, 1lème Edition, Besançon, France, (2014), pp. 76-81.

[24] P. Vrignat, J.F. Millet, F. Duculty, S. Begot and M. Avila, A technology shift for a fireworks controller, 2nd International Congress in Education, Innovation and Learning Technologies, 2015. 